Keywords: gestational trophoblastic neoplasia; methotrexate; drug resistance; neoplasm; prognosis; decision support techniques; chorionic gonadotropin

\title{
Early prediction of treatment resistance in low-risk gestational trophoblastic neoplasia using population kinetic modelling of hCG
} measurements

B You ${ }^{\star}, 1,2$, R Harvey ${ }^{3,4}$, E Henin ${ }^{1}$, H Mitchell ${ }^{3}$, F Golfier ${ }^{2}$, P M Savage ${ }^{3}$, M Tod ${ }^{1}$, M Wilbaux ${ }^{1}$, G Freyer ${ }^{1}$ and $\left.\mathrm{M} \mathrm{J} \mathrm{Seckl}\right|^{3}$

${ }^{1}$ EMR UBCL/HCL 3738, Université Claude Bernard Lyon-1, Hospices Civils de Lyon, Lyon, France; ${ }^{2}$ Centre de Référence des Maladies Trophoblastiques, Hospices Civils de Lyon, Lyon, France and ${ }^{3}$ Charing Cross Hospital Trophoblastic Disease Centre, London, UK

Background: In low-risk gestational trophoblastic neoplasia (GTN) patients, a predictive marker for early identification of methotrexate (MTX) resistance would be useful. We previously demonstrated that kinetic modelling of human chorionic gonadotrophin (hCG) measurements could provide such a marker. Here we validate this approach in a large independent patient cohort.

Methods: Serum hCG measurements of 800 low-risk GTN patients treated with MTX were analysed. The cohort was divided into Model and Test data sets. hCG kinetics were described from initial treatment day to day 50 using: '(hCG(time)) $=h C G 0^{*} \exp (-$ $k^{*}$ time) $+h C G r e s^{\prime}$, where hCGres is the modelled residual production, hCG0 is the baseline hCG level, and $k$ is the rate constant. HCGres-predictive value was investigated against previously reported predictors of MTX resistance.

Results: Declining hCG measurements were well fitted by the model. The best discriminator of MTX resistance in the Model data set was hCGres, categorised by an optimal cut-off value of $>20.44 \mathrm{IUI}^{-1}$ : receiver-operating characteristic (ROC) area under the curve $(\mathrm{AUC})=0.87 ; \mathrm{Se}=0.91 ; \mathrm{Sp}=0.83$. The predictive value of $\mathrm{hCG}$ res was reproducible using the Test data set: $\mathrm{ROC}$ $A U C=0.87 ; \mathrm{Se}=0.88 ; \mathrm{Sp}=0.86$. Multivariate analyses revealed $\mathrm{hCG}$ res as a better predictor of $\mathrm{MTX}$ resistance $(H R=1.01$, $P<0.0001)$ and MTX failure-free survival $(H R=13.25, P<0.0001)$ than other reported predictive factors.

Conclusion: hCGres, a modelled kinetic parameter calculated after fully dosed three MTX cycles, has a reproducible value for identifying patients with MTX resistance.

Human chorionic gonadotrophin (hCG) is a sensitive serological marker in monitoring trophoblastic disease activity in gestational trophoblastic disease (GTD; Bagshawe, 1992). Patients diagnosed with low-risk gestational trophoblastic neoplasias (GTN), comprising 95\% of malignant GTD forms (including invasive mole, choriocarcinoma, epitheliod trophoblastic and placental site trophoblastic tumours) have International Federation of Gynecology and Obstetrics (FIGO) 2000 risk scores ranging from 0 to 6 and are treated using single-agent chemotherapy (Kohorn, 2001; Chalouhi et al, 2009). The 8-day methotrexate (MTX) protocol modified by Bagshawe et al, 1989 is the most commonly used regimen in Europe (Savage et al, 2008; Chalouhi et al, 2009;

*Correspondence: Dr B You; E-mail: benoit.you@chu-lyon.fr

Previous presentations: 2011 ASCO Annual Meeting, IL, Chicago, USA (Poster Abstract number 5099); 2011 International Society for the Study of Trophoblastic Diseases Congress, Budapest, Hungary (Oral presentation).

${ }^{4}$ Richard Harvey is the joint first author.

Received 8 November 2012; revised 12 February 2013; accepted 19 February 2013; published online 16 April 2013

(c) 2013 Cancer Research UK. All rights reserved 0007-0920/13 
Kerkmeijer et al, 2009). MTX treatment is continued until either normalisation of serum hCG concentration and further three cycles or resistance to MTX therapy is detected (Seckl et al, 2010). In cases of MTX resistance, observed in nearly one-half of low-risk cases (Sita-Lumsden et al, 2012), patients are changed to either actinomycin D (ACT-D) or to a combination of etoposide, MTX, ACT-D, cyclophosphamide and vincristine regimen (EMA-CO) regimens (Powles et al, 2007; Patel \& Desai, 2010). Tumour chemoresistance is frequently defined as an increase or stagnation of hCG levels over a 2- to 3-week period, but no consensus guideline has been defined (Foulmann et al, 2006). Ambiguity concerning the definition of MTX resistance based on changing hCG levels increases the likelihood that patients will receive repetitive ineffective cycles of MTX chemotherapy.

Several previous studies have attempted to develop new methods to enable earlier identification of patients that will develop MTX resistance (van Trommel et al, 2006; Savage et al, 2008; Kerkmeijer et al, 2009; Growdon et al, 2009a). Among these, a previous study led by the French reference centre for treatment of GTN (Centre de Référence des Maladies Trophoblastiques, Lyon, France), showed that mathematical modelling of individual declining hCG measurements using a population kinetic approach is feasible, and that derived parameters were strong early predictors of MTX resistance (You et al, 2010). The study concluded that validation of the study's assumptions in independent cohorts of patients was warranted.

Charing Cross Hospital's Trophoblastic Disease Unit (London, UK) is a leading centre for treatment of GTN (Seckl et al, 2010). The objective of the present study was to validate the predictive value of the hCG kinetic modelling approach with respect to MTX resistance in low-risk GTN, using the Charing Cross Centre's data.

\section{PATIENTS AND METHODS}

Patient selection and hCG analysis. HCG measurement and clinical data from patients diagnosed with low-risk GTN and registered between August 1991 and January 2011 were retrieved from the Charing Cross Hospital's database. All patients were treated with the 8-day MTX regimen of $50 \mathrm{mg}$ intramuscular MTX on days $1,3,5$ and 7 , combined with $15 \mathrm{mg}$ oral folinic acid on days 2, 4, 6 and 8, every 2 weeks as first-line treatment for low-risk GTN according to the FIGO 2000 guidelines (Bagshawe et al, 1989; Kohorn, 2001). Treatment was continued for three cycles after normalisation of serum hCG concentration $\left(<5 \mathrm{IUl}^{-1}\right)$. In cases of MTX resistance, defined as three static or three rising hCG measurements, treatment was changed to second-line treatment (Seckl et al, 2010). The static versus changing distinction being $\pm 10 \%$ of the preceding serum hCG concentration.

Exclusion criteria included: (i) treatment with $<3$ cycles of MTX, (ii) treatment variants from the conventional MTX regimen and (iii) hCG monitoring with $<3$ measurement time points.

Total hCG serum concentrations were determined using an inhouse competitive radioimmunoassay, which uses a polyclonal rabbit anti-serum. The lower limit of detection is $1.6 \mathrm{IUl}^{-1}$ (calculated as 2 s.d. above the zero standard) and the coefficient of variation $(\mathrm{CV})$ at $\sim 3 \mathrm{IU}^{-1}$ is estimated at $14 \%$ (Harvey et al, 2010).

Model and test patient data sets. The 800 low-risk GTN patient cohort treated with the 8-day MTX regimen was divided into two: (i) a Model data set composed of 418 patients (195 MTX-resistant and 223 MTX-sensitive patients) and (ii) a Test data set comprising 382 patients (144 MTX-resistant and 238 MTXsensitive patients; Figure 1).

Procedure used to ascertain the predictive value of modelled kinetic parameters. A three-step scheme was used to ascertain the predictive value of population kinetic modelling of hCG data, using the patient cohort's Model and Test data sets (Figure 1) according to the following outline procedure:

- Estimation of individual kinetic parameters using population kinetic modelling of hCG measurements in Model data set patients. Receiver-operating characteristic (ROC) curves were used to assess the predictive values of the modelled kinetic parameters regarding MTX-resistance risk.

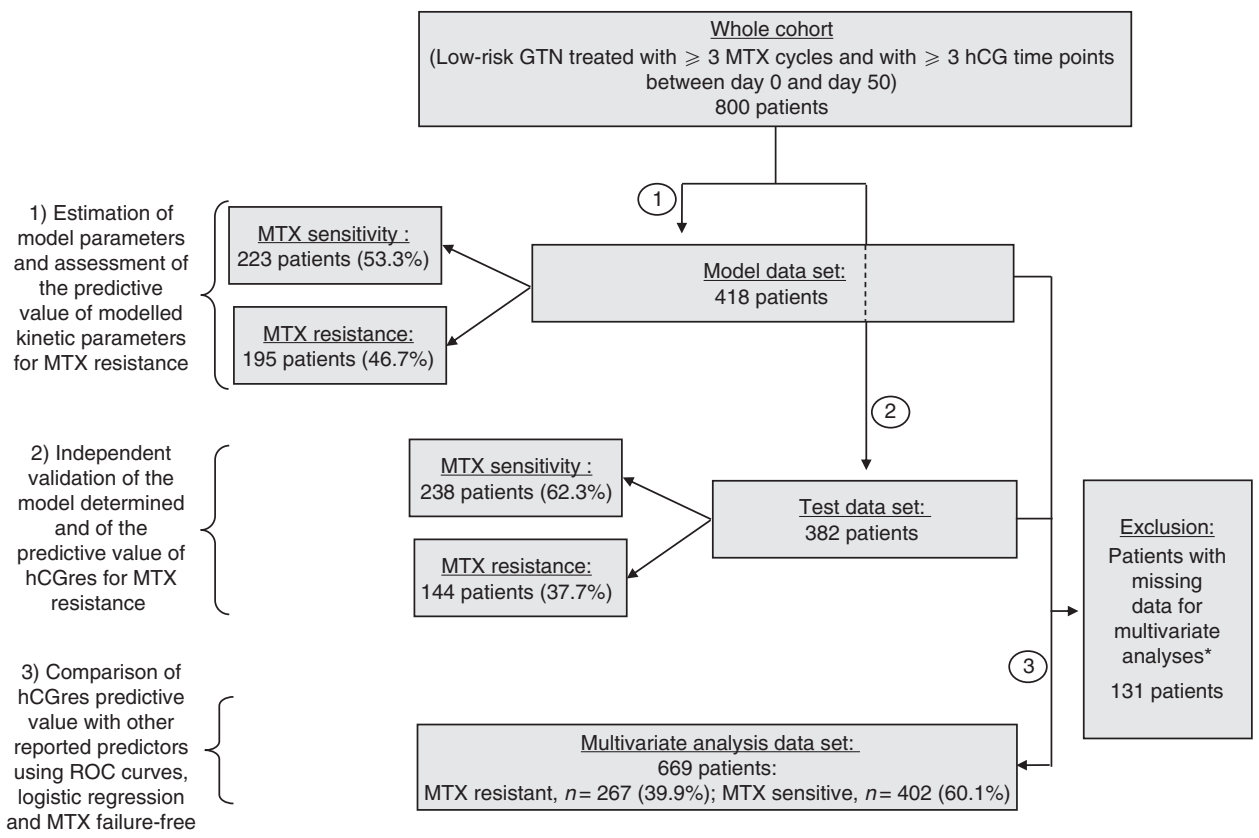

Figure 1. Flowchart of analysed patients in the three-time strategy. ${ }^{*} \mathrm{WHO}-\mathrm{FIGO}$ score (classified between 0 and 6 ); choriocarcinoma (yes vs no); hCG level measured 1 week after treatment start; hCG level in the seventh week. 
- Challenging the Test data set using the most predictive kinetic parameter identified, using the Model set to estimate the test's performance and assess reproducibility.

- Comparison of the relative predictive value of the best kinetic predictor identified using the Model data set with that of previously reported predictors of MTX resistance, using ROC curves and multivariate statistics (logistic regression; Cox-model survival test) in a combined data set, called the multivariate analysis data set, comprising model and test patients that had all required data (Figure 1 ).

Step 1: population kinetic modelling of hCG measurements in model data set patients. The population pharmacokinetic model developed by the French national centre in 2010 was used to fit declining hCG measurements from data of patients extracted from the Charing Cross Hospital database (You et al, 2010). Details of the mono-exponential model have been previously described (You et al, 2010). The model was further refined and the kinetic parameter 'hCGres', related to residual production at the end of hCG decline, was derived following fitting of declining hCG values using the final model:

$$
\mathrm{hCG}_{i j}(t)=\left(\mathrm{hCG}_{i}^{*} e^{-\mathrm{Ki}^{*} \mathrm{t}}+\mathrm{hCGres}_{i}\right)\left(1+\varepsilon 1_{i j}\right)
$$

where $\mathrm{hCG}_{i j}(t)$ is the $j$ th hCG measurement in patient $i$ at time $t$; hCGres $_{i}$ is the hCG residual production of patient $i ; \varepsilon 1_{i j}$ represents the proportional residual variability at the $j$ th measurement of patient $i$, which is assumed to follow a normal distribution with zero mean and $\sigma^{2}$ variance. $\mathrm{Ki}$ is the hCG decline rate constant. Parameters K and hCGres were assumed to vary between patients according to a normal log distribution; because of the heterogeneity in baseline hCG levels, a Box-Cox distribution was assumed for hCG0 in the population. Moreover, censored observations of hCG titres below the limit of quantification (i.e., those measurements recorded as $<2 \mathrm{IUl}^{-1}$ ) were handled using the so-called M3 method, which considers the likelihood of observations being below the quantifiable limit (Beal, 2001; Ahn et al, 2008; Bergstrand and Karlsson, 2009). The kinetic analysis used hCG measurements taken between the first day of MTX treatment (day 0 ) and day 50. If the treatment was altered during the first 50 treatments days, further hCG titres were not included in the analysis. The predictive performance of the final model was evaluated by visual predictive check (VPC) method, an approach commonly adopted for internal validation in modelling studies (Brendel et al, 2006; Dartois et al, 2007). The analysis was performed using a non-linear mixed-effects modelling strategy, implemented in NONMEM Version 7 (Beal et al, 2009) (full details in Appendix online).

Assessment of predictive value for MTX resistance using ROC curve analysis in model data set patients. Continuous ROC curve analyses were used to assess the predictive values of modelled $\mathrm{K}$ and hCGres in the Model data set. Among them, the hCGres kinetic parameter, which offered the highest discriminative value, based on the highest value of ROC area under the curve (AUC) was selected for further analyses. The optimal hCGres cut-off value for discriminating resistant $v s$ sensitive patients was determined by the intersection of minimal overlap of the true-positive and truenegative populations for the Model data set, that is, where sensitivity (Se) + specificity (Sp) was maximised.

Step 2: validation of 'hCGres' predictive ability using Test data set. The final model and optimal cut-off value derived using the Model data set was applied, to independently estimate individual values of hCGres in the Test data set patients. As previously, declining hCG values observed during the first 50 MTX treatment days were fitted using the Maximum A posteriori algorithm in NONMEMTM software. The theoretical MTX resistance risk of the Test data set patients was defined in every subject based on the Model set's defined cut-off value. The accuracy of prediction was then assessed using discontinuous ROC curve analysis.

Step 3: assessment of predictive value of hCGres against previously reported MTX resistance predictors. Other previously reported predictive factors for chemoresistance considered in the comparative analysis were: WHO-FIGO score (classified between 0 and 6); choriocarcinoma (yes vs no; You et al, 2010); hCG level measured 1 week after treatment start, categorised according to Growdon et al (2009b) cut-offvalue > $2000 \mathrm{IUl}^{-1}$ (Growdan et al, $2009 \mathrm{~b}$ ) or hCG level in the seventh week, categorised according to different thresholds, $\leqslant 520.24 v s>520.24 \mathrm{IUl}^{-1}$ (van Trommel et al, 2006); $\leqslant 500 v s>500 \mathrm{IU}^{-1}$ (Savage et al, 2008); and $\leqslant 737$ vs $>737 \mathrm{IUl}^{-1}$ (Kerkmeijer et al, 2009).

Using the multivariate analysis data set, the relative predictive performance of modelled hCGres was compared against the previously reported predictors using ROC curve analysis. Moreover, the independence of the predictive value of hCGres for MTX resistance was compared against these predictors, considered as continuous or categorised covariates, using multivariate logistic regression. A backward elimination procedure was used to select the independent predictive factors.

Predictive value of hCGres regarding MTX failure-free survival against previously reported predictors. The data set was also used to assess hCGres-predictive ability regarding MTX treatment failure. MTX failure was defined as MTX resistance or relapse of disease. Relapse was defined as recurrent disease requiring further chemotherapy marked by elevated hCG detected following previously successful chemotherapy (where hCG had been normalised for $\geqslant 6$ weeks). The predictive value of hCGres was assessed using univariate (log-rank test) and multivariate (Cox model) survival tests. A backward elimination procedure was used to select the independent predictive factors. All tests were performed with S-Plus (TIBCO Software Inc., Palo Alto, CA, USA) or SPSS (SPSS Inc., Chicago, IL, USA) using a two-sided $0.05 \alpha$-risk.

Landmark timepoint analyses. Landmark time point analyses with time threshold set at 50 days were performed to ensure the validity of results and lack of bias. As a result, ROC curve analyses and survival tests were performed with specific data sets, excluding patients treated with a second-line treatment before day 50. Results are shown in Appendix, (online only) because results were consistent with those obtained with all data sets.

\section{RESULTS}

Patient characteristics in model and test data sets are presented in Table 1.

Population kinetic modelling of hCG kinetics in model data set patients. Parameter estimates and their respective s.e. are reported in Table 2. Typical values and CV between patients of hCG0, $\mathrm{K}$ and hCGres were $14400 \mathrm{IUl}^{-1}$ (CV: $\left.152 \%\right), 0.169$ per day (CV: 306\%) and $24.7 \mathrm{IU}^{-1}$ (CV: $\left.35 \%\right)$, respectively. The goodness-of-fit plots show that individual hCG profiles were well fitted during the first 50 treatment days by the model (Figures $2 \mathrm{~A}$ and 3). The VPC method showed that the median, $5 \%$ and $95 \%$ of the observed hCG values over time were included within $95 \%$ confidence interval boundaries of the simulated median, 5\% and 95\% of hCG, in keeping with the good data fitting by the model (Figure 2B).

Prediction of MTX resistance using derived kinetic parameters in model data set patients. The abilities of hCGres and $\mathrm{K}$ for predicting MTX resistance were compared using ROC curves. On the basis of the AUC values, hCGres was a better predictor of MTX 


\begin{tabular}{|c|c|c|}
\hline & Model data set & Test data set \\
\hline & No. patients & No. patients \\
\hline Age (IQR): years & $31.2(26.1-36.1)$ & $30.9(26.1-35.6)$ \\
\hline $\begin{array}{l}\text { Interval between evacuation and } \\
\text { first MTX dosing (IQR): months }\end{array}$ & $1.9(1.3-2.9)$ & $1.9(1.4-3.2)^{a}$ \\
\hline Choriocarcinoma (\%) & $19(4.5)$ & $18(4.7)$ \\
\hline \multicolumn{3}{|l|}{ WHO-FIGO sore } \\
\hline $\begin{array}{l}0(\%) \\
1(\%) \\
2(\%) \\
3(\%) \\
4(\%) \\
5(\%) \\
6(\%)\end{array}$ & $\begin{array}{c}37(8.9) \\
76(18.2) \\
95(22.7) \\
105(25.1) \\
70(16.7) \\
28(6.7) \\
7(1.7)\end{array}$ & $\begin{array}{c}33(8.6) \\
73(19.1) \\
82(21.5) \\
108(28.3) \\
60(15.7) \\
18(4.7) \\
8(2.1)\end{array}$ \\
\hline $\begin{array}{l}\text { Number of MTX received: median } \\
\text { (IQR) (range) }\end{array}$ & $6(4-7)(3-14)$ & $6(4-7)(3-12)$ \\
\hline \multicolumn{3}{|l|}{ Response to MTX } \\
\hline $\begin{array}{l}\text { Sensitive (\%) } \\
\text { Resistant (\%) }\end{array}$ & $\begin{array}{l}223(53.3) \\
195(46.7)\end{array}$ & $\begin{array}{l}238(62.3) \\
144(37.7)\end{array}$ \\
\hline Total ( $n=800$ patients) & 418 & 382 \\
\hline \multicolumn{3}{|c|}{$\begin{array}{l}\text { Abbreviations: } I Q R=\text { interquartile range; } M T X=\text { methotrexate; } W H O-F I G O=W H O-I n t e r- \\
\text { national Federation of Gynecology and Obstetrics. } \\
\text { a Unknown for one patient. }\end{array}$} \\
\hline
\end{tabular}

\begin{tabular}{|c|c|c|c|c|}
\hline \multicolumn{5}{|c|}{$\begin{array}{l}\text { Table 2. Typical parameters and precision }{ }^{\text {a }} \text { of estimates for Model data } \\
\text { set patients ( } n=418 \text { patients) }\end{array}$} \\
\hline Parameter & $\begin{array}{l}\text { Population } \\
\text { value }\end{array}$ & $\begin{array}{c}\text { Median of } \\
\text { individual } \\
\text { predictions }\end{array}$ & $\begin{array}{l}\text { Interindividual } \\
\text { variability }\end{array}$ & $\begin{array}{l}\text { Residual } \\
\text { variability }\end{array}$ \\
\hline $\begin{array}{l}\text { hCG0 } \\
\left.(I U)^{-1}\right)^{b}\end{array}$ & 14400 (5.7\%) & 16574 & $152 \%$ & $34.4 \%$ (1.4\%) \\
\hline K (per day) & $0.169(1.3 \%)$ & 0.174 & $306 \%$ (6.7\%) & ND \\
\hline $\begin{array}{l}\text { hCGres } \\
\left(I U I^{-1}\right)\end{array}$ & 24.7 (11\%) & 21.97 & $35 \%(4.9 \%)$ & ND \\
\hline \multicolumn{5}{|c|}{ 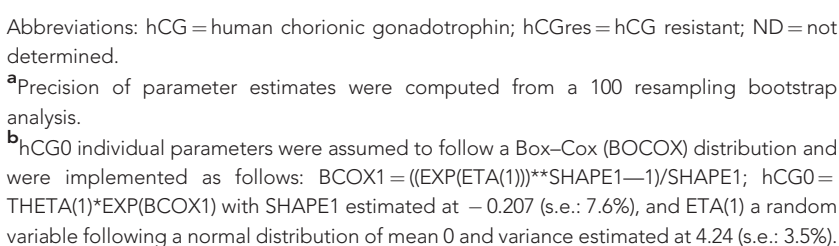 } \\
\hline
\end{tabular}

resistance than $\mathrm{K}$ (AUC for continuous $\mathrm{ROC}=0.94,95 \%$ $\mathrm{CI}=0.91-0.96$ vs $\mathrm{AUC}=0.69,95 \% \mathrm{CI}=0.64-0.74$; Figure $4 \mathrm{~A}$ ) and was, therefore, selected for further analyses. The optimal cutoff value for hCGres for discriminating resistant $v s$ sensitive predictor was found to be $20.44 \mathrm{IUl}^{-1}$. When the model data set patients were reassessed by discontinuous ROC analysis after classifying them into two predictive groups, as either high MTXresistant risk $\left(>20.44 \mathrm{IUl}^{-1}\right)$ or low risk $\left(\leqslant 20.44 \mathrm{IUl}^{-1}\right)$ to ascertain hCGres's predictive diagnostic performance at the optimal cut-off value, the AUC was $0.87(95 \% \mathrm{CI}=0.65-1)$ with $91 \%$ sensitivity $(95 \% \quad \mathrm{CI}=0.86-0.94), \quad 83 \%$ specificity $\quad(95 \%$ $\mathrm{CI}=0.78-0.88), 83 \%$ positive predictive value (PPV) and $91 \%$ negative predictive value (NPV).
Validation of hCGres-predictive ability using the test data set. Individual values of hCGres were estimated in Test data set patients using the same model as defined above. Patient risk of resistance was predicted based on the $>20.44 \mathrm{IUl}^{-1}$ hCGres threshold. The outcome regarding the predictive value of modelled hCGres in the Test patient data set was almost identical to that observed with the Model set patients: $\mathrm{AUC}=0.87,(95 \%=0.65-1)$ with $88 \%$ sensitivity $(95 \% \mathrm{CI}=0.82-0.93), 86 \%$ specificity, $(95 \%$ $\mathrm{CI}=0.81-0.90), \mathrm{PPV}=79 \%$ and $\mathrm{NPV}=92 \%$, confirming the high reproducibility of the methodological approach.

Comparison of hCGres against other predictors of MTX resistance in the combined multivariate analysis data set including 669 patients with required data

Logistic regression. Using a binomial logistic regression model, including the WHO-FIGO score $(\mathrm{HR}=1.17,95 \% \mathrm{CI}=0.99-1.38)$, choriocarcinoma (yes vs. no, $\mathrm{HR}=1.02,95 \% \mathrm{CI}=0.36-2.87$ ), high hCG titre at week 1 categorised by $>2000 \mathrm{IU}^{-1}$ cut-off value defined by Growdon et al (2009a; HR =1, 95\% CI =1-1), high hCG at week 7 categorised by $>737 \mathrm{IUl}^{-1}$ threshold defined by Kerkmeijer et al (2009; HR $=1,95 \% \mathrm{CI}=1-1.01)$ and hCGres, the only significant independent predictive factor of MTX resistance was hCGres $(\mathrm{HR}=1.009,95 \% \mathrm{CI}=1.007-1.011, P<0.0001)$.

ROC curve analyses. The AUCs of the discrete ROC curves for other previously reported predictors of the MTX resistance in multivariate analysis data set were also assessed (Figure 4B). The highest AUC was with hCGres (>20.44 $\mathrm{IUl}^{-1}$ : AUC $=0.86,95 \%$ $\mathrm{CI}=0.83-0.89)$ compared with hCG value on week 1 $\left(>2000 \mathrm{IUl}^{-1}: \mathrm{AUC}=0.64,95 \% \mathrm{CI}=0.60-0.69\right), \mathrm{hCG}$ values on week $7\left(>500 \mathrm{IUl}^{-1}: \mathrm{AUC}=0.70,95 \% \mathrm{CI}=0.65-0.74\right.$; $>520.24 \mathrm{IUl}^{-1}: \mathrm{AUC}=0.69,95 \% \mathrm{CI}=0.65-0.74 ;>737 \mathrm{IUl}^{-1}$ : $\mathrm{AUC}=0.66,95 \% \mathrm{CI}=0.62-0.70)$ and the WHO-FIGO score $(\mathrm{AUC}=0.66,95 \% \mathrm{CI}=0.62-0.70)$.

Prediction of MTX failure. After a median follow-up period of 4.89 years, 358 patients out of 800 patients (44.7\%) experienced resistance and/or a relapse (i.e., MTX treatment failure). Relapses were observed in 19 out of 461patients considered sensitive to MTX (4.1\%) after a median of 277 days (IQR: 187-462). All patients with MTX resistance or relapse were treated with secondline treatment (Table 3). Three-year MTX failure-free survival was significantly higher in patients with hCGres values $\leqslant 20.44,(88.4 \%$ vs $17 \%, P<0.0001$; Figure $4 \mathrm{C}$ ).

Data from 669 patients in the multivariate analysis data set was also analysed in a multivariate Cox model. The only significant independent predictors of MTX failure-free survival were as follows: high-modelled individual hCGres categorised by $>20.44$ threshold $(\mathrm{HR}=14.51,95 \% \mathrm{CI}=9.78-21.09)$; high hCG titre at week 1 categorised by $>2000 \mathrm{IUl}^{-1}$ cut-off value defined by Growdon et al (2009a; HR $=0.62,95 \% \mathrm{CI}=0.45-0.83)$ and high hCG at week 7 categorised by $>737 \mathrm{IUl}^{-1}$ threshold defined by Kerkmeijer et al (2009; HR $=2.19,95 \% \mathrm{CI}=1.65-2.90)$.

\section{DISCUSSION}

Current trophoblastic disease management is heavily reliant on serum hCG monitoring (Seckl et al, 2010). As such, hCG and lowrisk GTN provides an excellent disease model to demonstrate the potential utility of population kinetic modelling of disease-specific biomarkers for rationalisation of treatment decisions.

Using the largest cohort of low-risk GTN patients reported in the literature to date, we have demonstrated that population kinetic modelling of patients' hCG measurements during the first 50 days of MTX treatment provides a parameter, hCGres, which is a strong, independent and reproducible predictor for MTX resistance. 

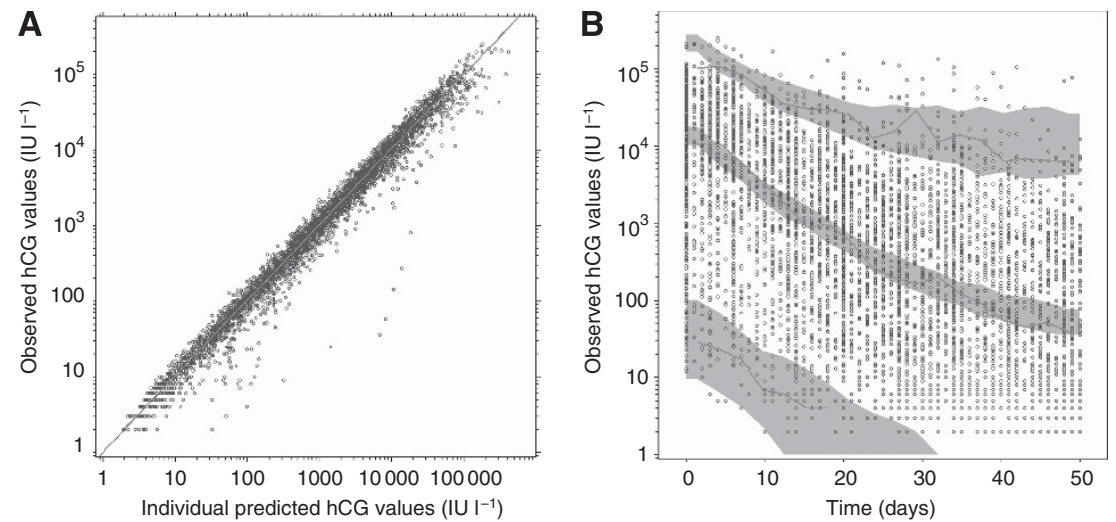

Figure 2. Internal qualification of the model in Model data set patients ( $n=418$ patients). (A) Observed vs individual predicted hCG titres (blue circles), identity line (black line), smooth line of the cloud of points (red line). (B) Visual predictive checks of hCG concentrations. The observations are symbolised by the blue circles, and the median, $5 \%$ and $95 \%$ of observations are represented by red lines. Blue areas represent the $95 \%$ prediction interval for the median, $5 \%$ and $95 \%$, computed from 1000 simulated replicates. A full colour version of this figure is available at the British Journal of Cancer journal online.
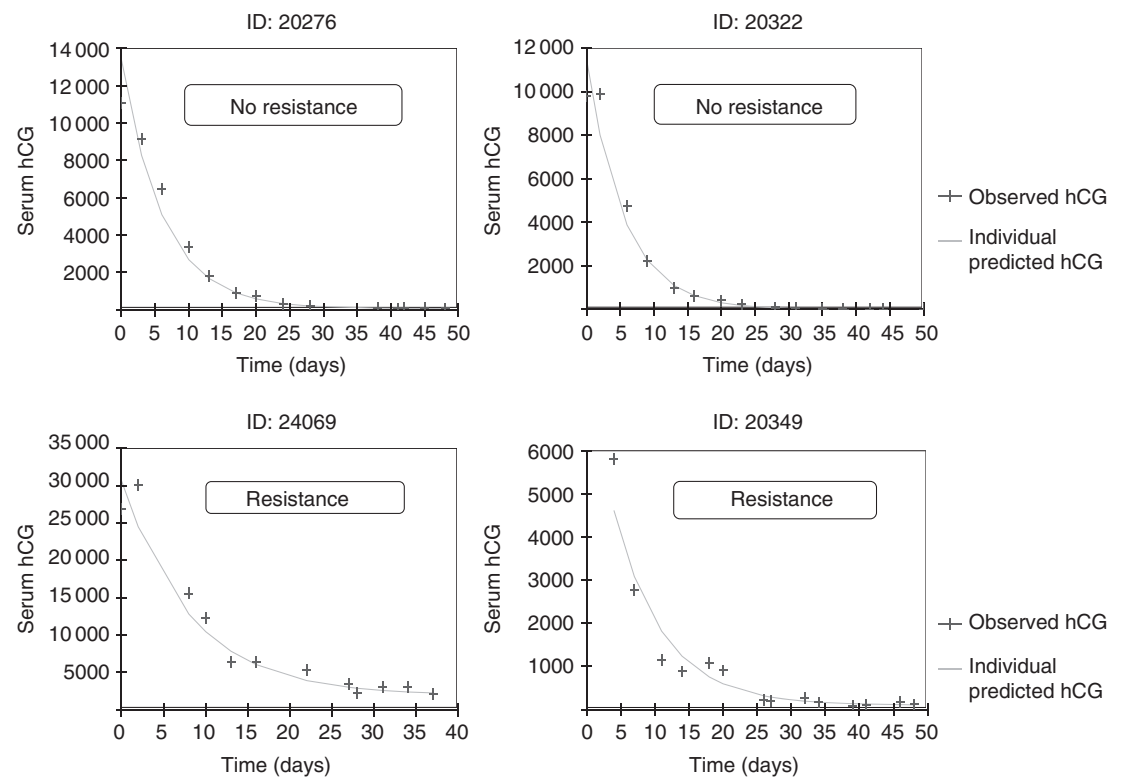

Figure 3. Examples of four typical patients of Model data set. The blue dots are the observed hCG values while the green line represents the individual predicted values. Resistances to MTX are specified. A full colour version of this figure is available at the British Journal of Cancer journal online.

The continuous ROC AUC for discriminating subsequent MTX resistance in the Model data set was 0.94, which, according to Swets' (1988) criteria, describes an 'excellent' test. These findings are consistent with those of the first French study, and those of a recent retrospective analysis of American GOG-174 phase III trial data (You et al, 2010; You et al, 2012). Moreover, the risk of MTX resistance could be predicted with better accuracy than using other previously reported predictors, including absolute hCG concentration thresholds. The kinetic approach was expected to have greater predictive power and show better reproducibility than using single hCG concentration thresholds as the effects of measurement intra- or interindividual inaccuracies (e.g., related to the use of different immunoassays) are reduced during fitting by the least squares method across multiple measurements (You et al, 2010; Bidart et al, 1999).

However, the application and comparability of the cut-off values derived by the other studies is limited due to differences in (i) study design and categorisation of patients (Kerkmeijer et al (2009) study's derived cut-off value was for discrimination of 'monotherapy resistance' rather than just MTX resistance alone), (ii) prevalence of the test positive condition and (iii) the use of different assays (van Trommel et al, 2006; Savage et al, 2008).
The proportion of MTX-resistant patients in our low-risk GTN cohort (42\%) was higher than the varying proportions reported in earlier clinical studies (ranging 18 to 40\%; Powles et al, 2007; Patel and Desai, 2010), but is in agreement with the largest studies which found 39-43\% (Kerkmeijer et al, 2009; Sita-Lumsden et al, 2012).

Most strikingly, when the same cut-off value derived for hCGres in the present study was applied to the original French study's data set of 154 patients, where serum hCG was measured weekly using various assays at different centres, an equivalent ROC assessment for MTX resistance was found with $\mathrm{AUC}=0.85(95 \% \mathrm{CI}=0.78-$ 0.92 ), further confirming the high reproducibility of the methodological approach.

The hCGres parameter also appeared to be a strong independent predictor of MTX failure, defined as MTX resistance or relapse, sometimes occurring several months after hCG normalisation. Prediction of MTX failure could have greater clinical relevance than MTX resistance alone, as it may be argued that the better measure of a specific treatment's success is lifelong cure from the disease (Powles et al, 2007). Patients with an hCGres $>20.44 \mathrm{IU}^{-1}$ after three fully dosed treatment cycles of MTX had 

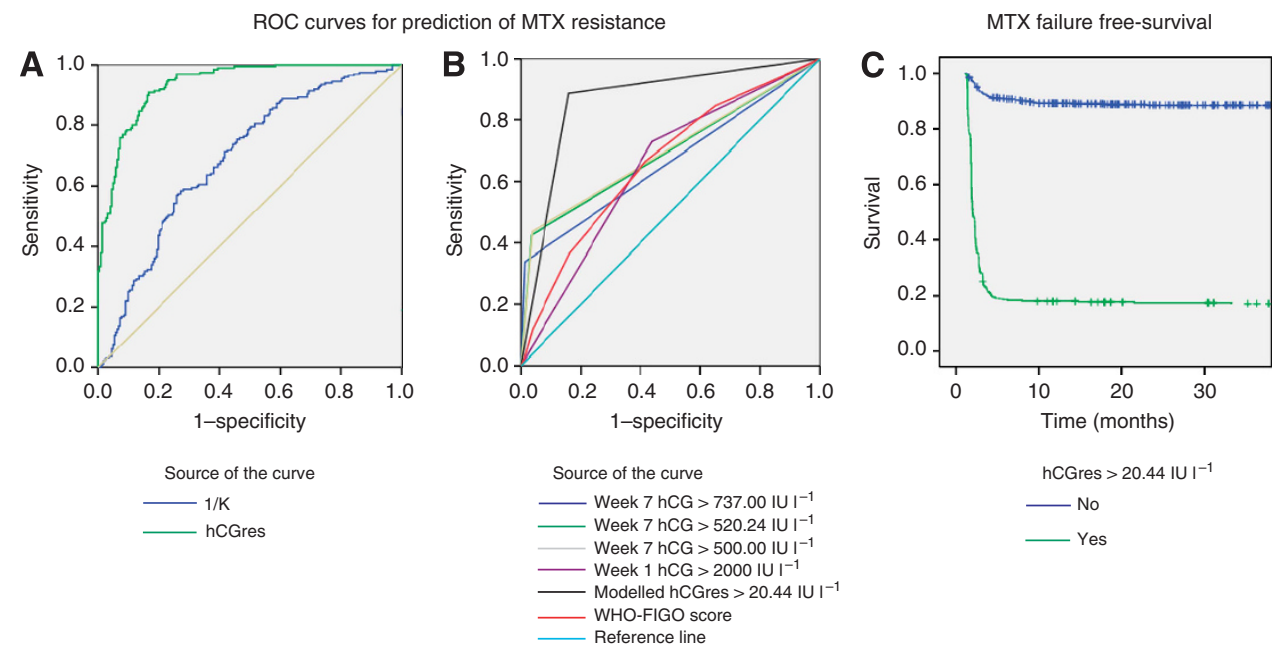

Figure 4. Predictive value of modelled kinetic parameters. (A) Predictions of MTX resistance offered by two modelled kinetic parameters $\mathrm{K}$ $(A \cup C=0.69,95 \% \mathrm{Cl}=0.64-0.74)$ and hCGres (AUC $=0.94,95 \% \mathrm{Cl}=0.91-0.96)$. (B) Predictive values of hCGres $\left(>20.44 \mathrm{IUI} \mathrm{I}^{-1}: \mathrm{AUC}=0.86,95 \%\right.$ $\mathrm{Cl}=0.83-0.89$ ) against previously reported predictors: hCG values on week 7 (>737 IUI ${ }^{-1}: A U C=0.66 ; 95 \% \mathrm{Cl}^{2}=0.62-0.70 ;>520.24 \mathrm{IU} \mathrm{I}^{-1}$ : $\left.A \cup C=0.69,95 \% \mathrm{Cl}=0.65-0.74 ;>500 \mathrm{IUI}^{-1}: \mathrm{AUC}=0.70,95 \% \mathrm{Cl}=0.65-0.74\right)$ and the WHO-FIGO score $(\mathrm{AUC}=0.66,95 \% \mathrm{Cl}=0.62-0.70)$. (C) Predictive value of modelled hCGres categorised by 20.44 cut-off value regarding MTX failure-free survival. Three-year MTX failure-free survival $=89.7 \%$ vs $21.8 \%$, hCGres $<0.0001$.

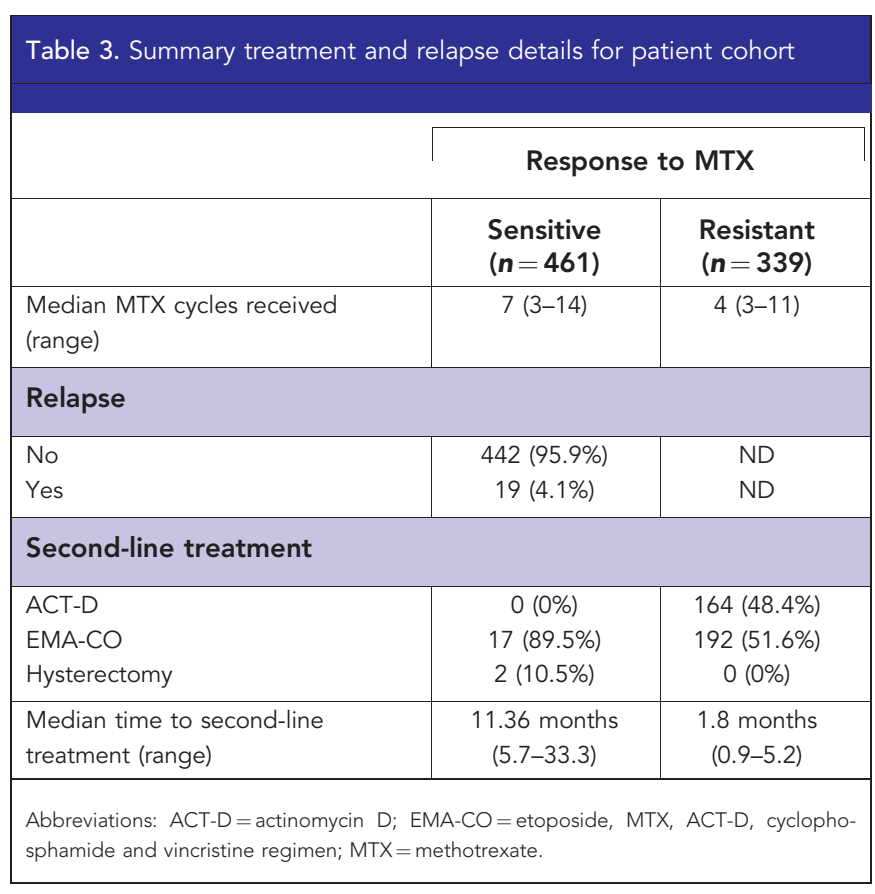

a more than $80 \%$ chance of MTX-resistant disease and a $92 \%$ 3-year MTX treatment failure risk.

Our findings suggest a possible rationale to consider changing to second-line treatment immediately following prediction of MTX resistance using the kinetic method. In the present data set, if patients with hCGres values $>20.44 \mathrm{IUl}^{-1}$ were changed to second-line treatment after only three fully dosed cycles of MTX treatment, a total of 478 MTX cycles administered to 226 patients, who were later determined to be MTX resistant and treated with ACT-D $(n=109)$ or EMA-CO $(n=117)$ regimens, would have been saved ( $83 \%$ truepositive rate). Such a strategy would reduce the duration of MTX therapy by 2 weeks in $50 \%$ patients (and by 4 weeks in $25 \%$ patients) and limit unnecessary use of MTX. However, this approach would also have led to unnecessary treatment change in 66 patients who, despite their high-modelled hCGres values, were cured with MTX therapy ( $17 \%$ false-positive rate). The strategy appears a fair one as only 9 out of 66 (14\%, or $1 \%$, of the entire cohort) of these patients, whose hCG levels where $\geqslant 300 \mathrm{IUl}^{-1}$ after three courses of MTX, would, according to current clinical practice, have been switched to the higher toxicity EMA-CO regimen (Seckl et al, 2010), with minimal probability of mortality as recently shown (Alifrangis et al, 2013). The remaining 57 patients would have been changed to the lower toxicity ACT-D regimen, expected to be highly curative in such patients, as shown by the GOG-174 trial outcomes (Osborne et al, 2011). Although unnecessary treatment with EMA-CO represents a greater clinical risk than prolonged ineffective treatment with MTX, where the agreeable balance between them exists is a matter for further research. The authors acknowledge that implementation of a strategy requiring weekly measurements of serum hCG titres may not be possible in all parts of the world.

In summary, we have shown that of the previously reported efforts to utilise weekly hCG measurements to predict MTX resistance in low-risk GTN, the population kinetic modelling approach had the highest predictive power and was the most reproducible. We have shown that by using individual calculated hCGres values, it was possible to accurately identify after only three fully dosed MTX cycles, $\sim 80 \%$ of patients who will develop MTX resistance. Although model building is a complex process, our results may have practical clinical application. An online programme integrating the model could easily calculate individual modelled kinetic parameters, such as hCGres, from a patient's hCG results. We are currently designing a prospective multicentre European clinical trial with the European Organization for Treatment of Trophoblastic Disease, which would employ such a programme to further validate the clinical utility of hCGres for predicting MTX resistance, particularly with regard to reproducibility of predictive performance, inter-assay hCG cut-off values and the minimum interval requirement for testing.

\section{ACKNOWLEDGEMENTS}

MJS and RH are supported by the Imperial Experimental Cancer Medicine Centre and by the Biomedical Research Centre grants from Cancer Research UK, the UK Department of Health and National Institute for Health Research. 


\section{CONFLICT OF INTEREST}

The authors declare no conflict of interest.

\section{REFERENCES}

Ahn JE, Karlsson MO, Dunne A, Ludden TM (2008) Likelihood based approaches to handling data below the quantification limit using NONMEM VI. J Pharmacokinet Pharmacodyn 35: 401-421.

Alifrangis C, Agarwal R, Short D, Fisher RA, Sebire NJ, Harvey R, Savage PM, Seckl MJ (2013) EMA/CO for high-risk gestational trophoblastic neoplasia: good outcomes with induction low-dose etoposide-cisplatin and genetic analysis. J Clin Oncol 31: 280-286.

Bagshawe KD (1992) Choriocarcinoma. A model for tumour markers. Acta Oncol 31: 99-106.

Bagshawe KD, Dent J, Newlands ES, Begent RH, Rustin GJ (1989) The role of low-dose methotrexate and folinic acid in gestational trophoblastic tumours (GTT). Br J Obstet Gynaecol 96: 795-802.

Beal SL (2001) Ways to fit a PK model with some data below the quantification limit. J Pharmacokinet Pharmacodyn 28: 481-504.

Beal SL, Sheiner LB, Boeckmann A, Bauer RJ (2009) NONMEM User's Guides (1989-2009). Icon: Ellicott City, MD, USA.

Bergstrand M, Karlsson MO (2009) Handling data below the limit of quantification in mixed effect models. Aaps J 11: 371-380.

Bidart JM, Thuillier F, Augereau C, Chalas J, Daver A, Jacob N, Labrousse F, Voitot H (1999) Kinetics of serum tumor marker concentrations and usefulness in clinical monitoring. Clin Chem 45: 1695-1707.

Brendel K, Comets E, Laffont C, Laveille C, Mentre F (2006) Metrics for external model evaluation with an application to the population pharmacokinetics of gliclazide. Pharm Res 23: 2036-2049.

Chalouhi GE, Golfier F, Soignon P, Massardier J, Guastalla JP, Trillet-Lenoir V, Schott AM, Raudrant D (2009) Methotrexate for 2000 FIGO low-risk gestational trophoblastic neoplasia patients: efficacy and toxicity. Am J Obstet Gynecol 200: 643-646.

Dartois C, Brendel K, Comets E, Laffont CM, Laveille C, Tranchand B, Mentre F, Lemenuel-Diot A, Girard P (2007) Overview of model-building strategies in population PK/PD analyses: 2002-2004 literature survey 1. Br J Clin Pharmacol 64: 603-612.

Foulmann K, Guastalla JP, Caminet N, Trillet-Lenoir V, Raudrant D, Golfier F, Schott AM (2006) What is the best protocol of single-agent methotrexate chemotherapy in nonmetastatic or low-risk metastatic gestational trophoblastic tumors? A review of the evidence. Gynecol Oncol 102: $103-110$.

Growdon WB, Wolfberg AJ, Goldstein DP, Feltmate CM, Chinchilla ME, Lieberman ES, Berkowitz RS (2009a) Evaluating methotrexate treatment in patients with low-risk postmolar gestational trophoblastic neoplasia. Gynecol Oncol 112: 353-357.

Growdon WB, Wolfberg AJ, Goldstein DP, Feltmate CM, Chinchilla ME, Lieberman ES, Berkowitz RS (2009b) Evaluating methotrexate treatment in patients with low-risk postmolar gestational trophoblastic neoplasia. Gynecol Oncol 112: 353-357.

Harvey RA, Mitchell HD, Stenman UH, Blankenstein MA, Nustad K, Stieber P, Stewart W, Savage PM, Seckl MJ, Braunstein GD (2010) Differences in total human chorionic gonadotropin immunoassay analytical specificity and ability to measure human chorionic gonadotropin in gestational trophoblastic disease and germ cell tumors. J Reprod Med 55: 285-295.

Jonsson EN, Karlsson MO (1999) Xpose-an S-PLUS based population pharmacokinetic/pharmacodynamic model building aid for NONMEM. Comput Methods Programs Biomed 58: 51-64.

Kerkmeijer LG, Thomas CM, Harvey R, Sweep FC, Mitchell H, Massuger LF, Seckl MJ (2009) External validation of serum hCG cutoff levels for prediction of resistance to single-agent chemotherapy in patients with persistent trophoblastic disease. Br J Cancer 100: 979-984.

Kohorn EI (2001) The new FIGO 2000 staging and risk factor scoring system for gestational trophoblastic disease: description and critical assessment. Int J Gynecol Cancer 11: 73-77.

Lindbom L, Ribbing J, Jonsson EN (2004) Perl-speaks-NONMEM (PsN)-a Perl module for NONMEM related programming. Comput Methods Programs Biomed 75: 85-94.

Osborne RJ, Filiaci V, Schink JC, Mannel RS, Alvarez Secord A, Kelley JL, Provencher D, Scott Miller D, Covens AL, Lage JM (2011) Phase III trial of weekly methotrexate or pulsed dactinomycin for low-risk gestational trophoblastic neoplasia: a gynecologic oncology group study. J Clin Oncol 29: 825-831.

Patel SM, Desai A (2010) Management of drug resistant gestational trophoblastic neoplasia. J Reprod Med 55: 296-300.

Powles T, Savage PM, Stebbing J, Short D, Young A, Bower M, Pappin C, Schmid P, Seckl MJ (2007) A comparison of patients with relapsed and chemo-refractory gestational trophoblastic neoplasia. $\mathrm{Br} J$ Cancer $\mathbf{9 6}$ : 732-737.

Savage P, Seckl M, Short D (2008) Practical issues in the management of lowrisk gestational trophoblast tumors. J Reprod Med 53: 774-780.

Seckl MJ, Sebire NJ, Berkowitz RS (2010) Gestational trophoblastic disease. Lancet 376: 717-729.

Sita-Lumsden A, Short D, Lindsay I, Sebire NJ, Adjogatse D, Seckl MJ, Savage PM (2012) Treatment outcomes for 618 women with gestational trophoblastic tumours following a molar pregnancy at the Charing Cross Hospital, 2000-2009. Br J Cancer 107: 1810-1814.

Swets JA (1988) Measuring the accuracy of diagnostic systems. Science 240: 1285-1293.

The R Development CT (2010) R: A Language and Environment for Statistical Computing. R2120 edR Foundation for Statistical Computing: Vienna, Austria.

van Trommel NE, Massuger LF, Schijf CP, ten Kate-Booij MJ, Sweep FC, Thomas CM (2006) Early identification of resistance to first-line singleagent methotrexate in patients with persistent trophoblastic disease. J Clin Oncol 24: 52-58.

You B, Deng W, Oza A, Osborne B (2012) Validation of the predictive value of modeled hCG residual production "P" in low-risk gestational trophoblastic neoplasia (GTN) patients treated in GOG-174 phase III trial. Proceedings of the 2012 ASCO Annual Meeting. Abs 5110.

You B, Pollet-Villard M, Fronton L, Labrousse C, Schott AM, Hajri T, Girard P, Freyer G, Tod M, Tranchand B, Colomban O, Ribba B, Raudrant D, Massardier J, Chabaud S, Golfier F (2010) Predictive values of hCG clearance for risk of methotrexate resistance in low-risk gestational trophoblastic neoplasias. Ann Oncol 21: 1643-1650.

This work is published under the standard license to publish agreement. After 12 months the work will become freely available and the license terms will switch to a Creative Commons AttributionNonCommercial-Share Alike 3.0 Unported License.

Supplementary Information accompanies this paper on British Journal of Cancer website (http://www.nature.com/bjc) 